\title{
Tecnura
}

ESTUDIO DE CASO

\section{Aplicación de herramientas CAD/CAM para el diseño y fabricación de prototipos de moldes de inyección de plásticos}

\section{Tool application CAD / CAM for design and construction of a prototype of plastic injection mold}

\author{
Albert Miyer Suárez Castrillón¹, Wilson Tafur Preciado², Pedro Rodolfo Calderón Nieves ${ }^{3}$
}

Fecha de recepción: 2 de abril de 2015

Fecha de aceptación: 24 de agosto de 2015

Citar como: Suárez Castrillón, A. M., Tafur Preciado, W., \& Calderón Nieves, P. R. (2015). Aplicación de herramientas CAD/CAM para el diseño y fabricación de prototipos de moldes de inyección de plásticos. Revista Tecnura, 19(46), 115-121. doi:10.14483/udistrital.jour.tecnura.2015.4.a09

\section{Resumen}

El estudio, desarrollo y fabricación de moldes de inyección se facilita con la implementación de las herramientas CAD y CAM que ofrece el mercado, aplicando estas herramientas se diseñó y construyó un prototipo de molde para la inyección de materiales termoplásticos, con base en un molde del laboratorio de inyección de la Universidad de Pamplona, al cual se le hicieron modificaciones para experimentar con su diseño. Se fabricó el prototipo por medio de un escaneo 3D del molde original para obtener los archivos CAD /CAM con la simulación de la técnica de impresión 3D, para realizar un estudio experimental con el prototipo para la adaptación de sensores de temperatura y presión, y para recibir recubrimientos de materiales metálicos para su utilización en el proceso de moldeo por inyección.

Palabras clave: CAD, CAM, Impresión 3D, Molde de inyección, Moldeo por inyección.

\begin{abstract}
The study, development and production of injection molds comes with the implementation of CAD and CAM tools available on the market; using these tools, a prototype injection mold for thermoplastic materials was designed and built, based on a mold is injection in the laboratory of the University of Pamplona, in which a couple of modifications were made in order to experiment with its design. The prototype was manufactured through a 3D scan of the original mold to provide the CAD / CAM files with the simulated 3D printing technique for performing an experimental study with the prototype for adjusting temperature and pressure sensors and for coatings metallic materials for use in the injection molding process.
\end{abstract}

Keywords: 3D Printing, CAD, CAM, injection mold, injection molding.

\footnotetext{
Ingeniero Mecánico, doctor en Tecnologías Avanzadas de Producción. Docente de la Universidad de Pamplona. Pamplona, Colombia. Contacto: albertmiyer@unipamplona.edu.co

2 Ingeniero Mecánico, candidato a doctor en Ingeniería Mecánica. Docente de la Universidad de Pamplona. Pamplona, Colombia. Contacto: wilson.tafur@unipamplona.edu.co

Ingeniero Mecánico, Msc(c) en Ingeniería Mecánica. Docente de la Universidad de Pamplona. Pamplona, Colombia. Contacto: pedrocaldenie92@unipamplona.edu.co
} 


\section{INTRODUCCIÓN}

En el ámbito global es de gran relevancia y de constante análisis la industria de los plásticos y, por consiguiente, el proceso de moldeo por inyección, que es el más común en el procesamiento de piezas creadas en materiales termoplásticos. Debido a esto, se hace necesario investigar en el ámbito del desarrollo de moldes de inyección de manera rápida, eficiente y económica, permitiendo la reducción en los costos de inyección, ya que cada vez son más complejas las formas de los artículos fabricados por este medio (Dimitrov \& Saxer, 2012). En cuanto a las investigaciones en el campo del procesamiento de los parámetros de inyección, se han realizado varias investigaciones alrededor del mundo. Entre las más importantes está la de Xuan-Phuong (2014), en la cual se desarrolla un marco general para la optimización del proceso de moldeo por inyección de plásticos, contemplando una serie de puntos para lograr un óptimo desempeño de la máquina inyectora mediante métodos de análisis del proceso aplicando redes neuronales y algoritmo genético. Por su parte, el trabajo de Mustafa, Usuf, \& Kamber (2010) arrojó resultados sobre cómo la calidad de las piezas inyectadas son influenciadas por las variables de inyección.

En cuanto a la creación de moldes de inyección, existen varias técnicas, principalmente los de maquinado a alta velocidad $y$, recientemente, la impresión 3D (Hugo, Binayak, Wei, \& Ahna, 2014; Joanna, Karl, \& David, 2014). Cabe destacar que estos estudios han contado con las tecnologías CAD/CAM, con las cuales se facilita el diseño y simulación del proceso de inyección de plásticos (Alan \& Nguyen, 2014). Hay diferentes programas para este fin, entre ellos: C-mold, Mold Flow de Autodesk y Mold Works (SolidWork), los cuales permiten diseñar, simular y optimizar el proceso de inyección, y además permiten observar el comportamiento del molde sometido a las condiciones reales del proceso (Ching, Ming-Tsan , \& Yun-Hsiang, 2009).
La fabricación de moldes de inyección es algo casi artesanal, pues quien está encargado de hacerlo cuenta con conocimientos empíricos que por lo general son adquiridos por el método de prueba y error (Alvaro \& Gabriel, 2012), lo que supone pérdidas en todos los campos que involucren el desarrollo del molde. La utilización de software reduce la utilización del método de prueba y error, y consecuentemente la pérdida de material y los costos elevados, además reduce los tiempos de fabricación (Satoshi \& Shinji, 2014). En general, se puede realizar un completo estudio del molde y de las propiedades en la etapa de diseño antes de llegar a la etapa de fabricación (Eghbal \& Abu Bakar, 2012).

A continuación se describe la creación de un molde de inyección, por medio de impresión 3D, que se realizó en una máquina Next Engine Escaner3D, con la colaboración del Tecno-parque del SENA Nodo Ocaña, para dar solución a los problemas de diseño, tiempo y costos de fabricación de un molde para el proceso de inyección. Se utilizó la inyectora de la Universidad de Pamplona, se incorporaron sensores para el análisis de variables de inyección y estudios sobre la deposición de materiales metálicos como superficies de refuerzo. El material de impresión es termoplástico, sus propiedades no son las adecuadas para procesos de larga duración y a la vez proporcionar datos sobre el estudio de esta nueva técnica de fabricación.

\section{METODOLOGÍA}

Para llevar a cabo la investigación, el desarrollo del diseño y fabricación del prototipo, se tomó como base el molde de inyección de plásticos para hebillas (figura 1) que se tiene en el laboratorio de plásticos y mecanizado de la Universidad de Pamplona, el cual fue conducido hasta las instalaciones del Tecno-parque del SENA en Ocaña, donde se realizó el estudio y desarrollo del molde.

Para la fabricación del molde se siguieron varias etapas desde el desmonte hasta el escaneo de partes.

Luego del análisis del molde en las instalaciones del Tecno-parque, se procedió a realizar el diseño 


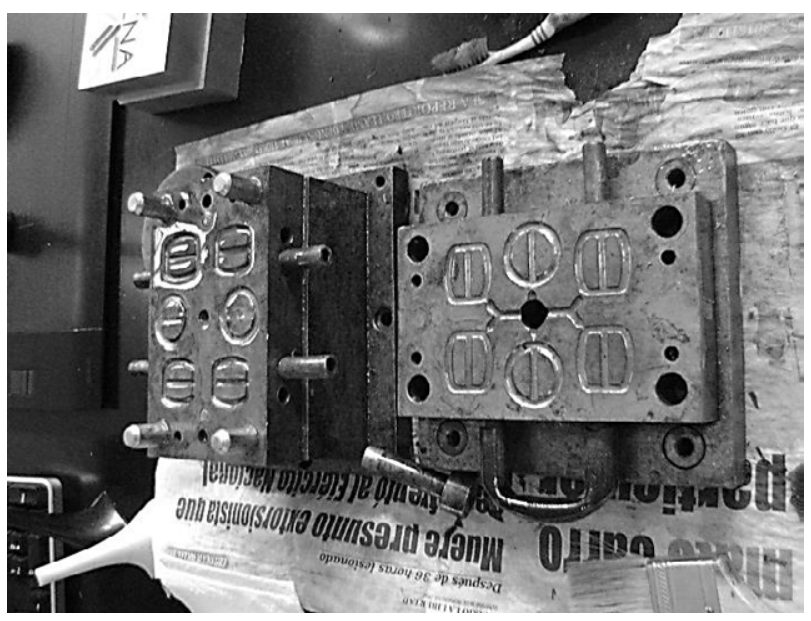

Figura 1. Molde de inyección.

Fuente: Elaboración propia

del nuevo molde prototipo que sería el modelo final a través de reingeniería. Es necesario aclarar que no todas las etapas Ilegaron a un feliz término, pero sí aportaron al desarrollo de la investigación para que no se afectaran las etapas que seguían ni llegaran a alterar los resultados que se esperaban.

\section{CAD (diseño asistido por computador)}

En esta etapa del proceso se llevaron a cabo los diseños en SolidWorks de cada una de las partes del molde de inyección, basándose en la información ya recopilada y obteniendo así los resultados esperados con el solo escaneo de las piezas. Esta operación resultó más complicada de lo esperado y al final se optó por la vía del CAD.

En la figura 2 se pueden observar algunas de las piezas elaboradas en esta etapa del proyecto.

Las cavidades del molde no representan hebillas como en el molde original, por el contrario, se decidió realizar cavidades en forma de probetas para el ensayo de tracción, aunque no con las medidas estándar, pues se tenía la limitación del tamaño de la impresión que realiza la máquina de impresión 3D. Por último se tiene el ensamblaje del molde con cada uno de sus componentes (figura 3).

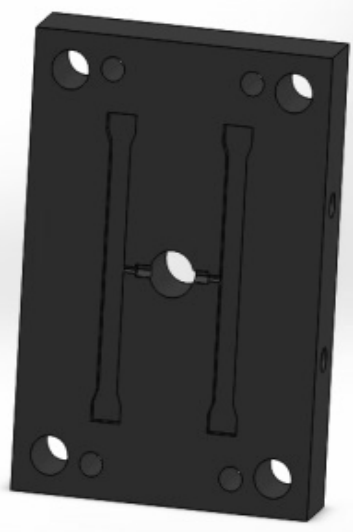

Figura 2. Placas del molde realizadas en SolidWorks.

Fuente: Elaboración propia

\section{CAM (manufactura asistida por computador)}

Luego de tener los archivos en CAD, con las especificaciones determinadas, se procedió con el último paso, que era imprimir las piezas, proceso para el cual tuvieron en cuenta los siguientes aspectos:

- Tamaño de las piezas $18 \times 18 \times 50$

- Material a utilizar

- Tiempos de impresión

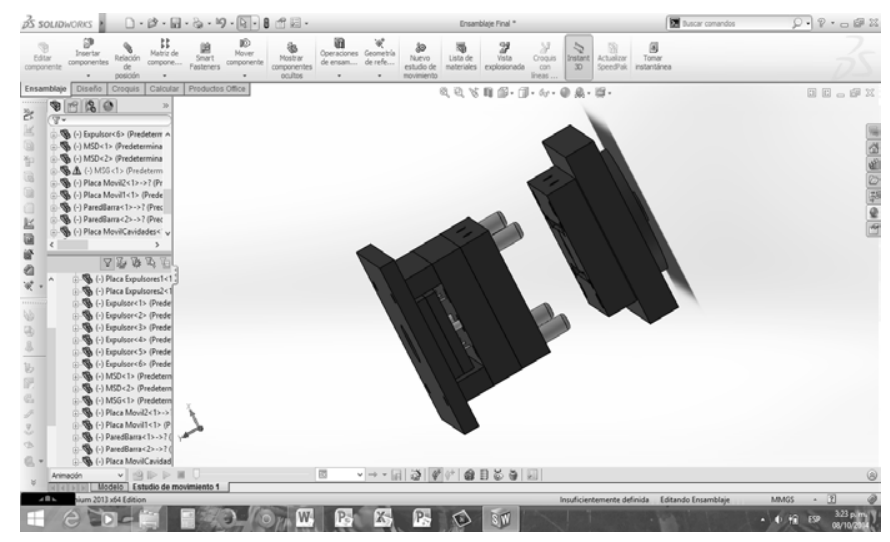

Figura 3. Molde de Inyección en CAD

Fuente: Elaboración propia 
Un aspecto importante consistió en no realizar al tamaño real las cavidades de las probetas para reducir el tamaño del molde y de igual forma ahorrar material. Se realizó un vaciado a las piezas que se seleccionaron para imprimir, transformándolas en cascarones que se podían unir. Para la labor de impresión se utilizó una impresora marca Dimension Elite, cuyo material de trabajo es el ABS (acrinilo butadieno estireno).

Mediante la interfaz del programa de la impresora se ajustó la pieza a las condiciones requeridas.
El archivo realizado en el software de diseño se guardó como .STL, para poder trabajarlo en el software de la impresora. En la figura 4 se muestra la interface de simulación.

Se seleccionaron las dos placas que poseían las cavidades del molde para ser impresas. Luego de realizar los vaciados, se imprimieron cuatro partes. Esta operación duró un tiempo promedio de 4,5 h por pieza (figura 5).

Una vez terminado el proceso de impresión se procedió a limpiar la pieza del material de soporte

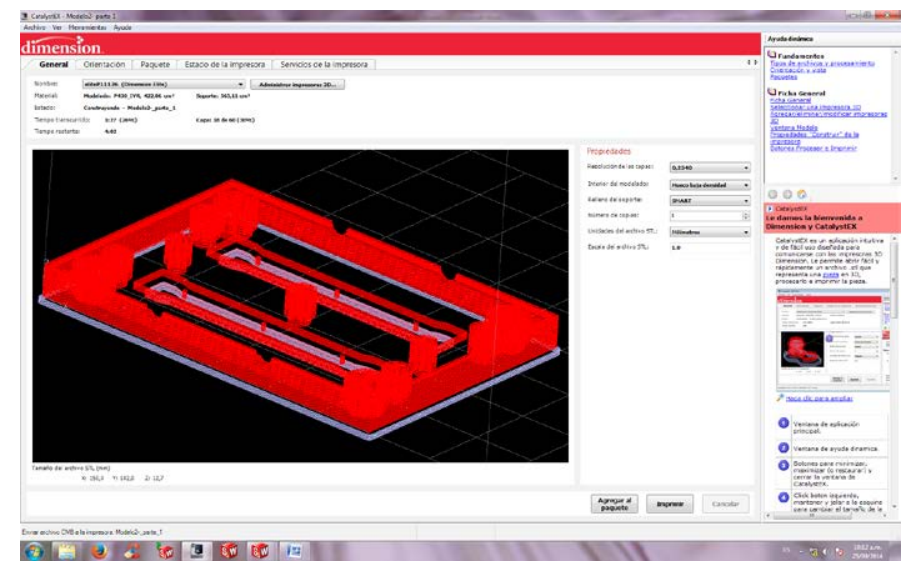

Figura 4. Simulación del proceso de impresión.

Fuente: Elaboración propia
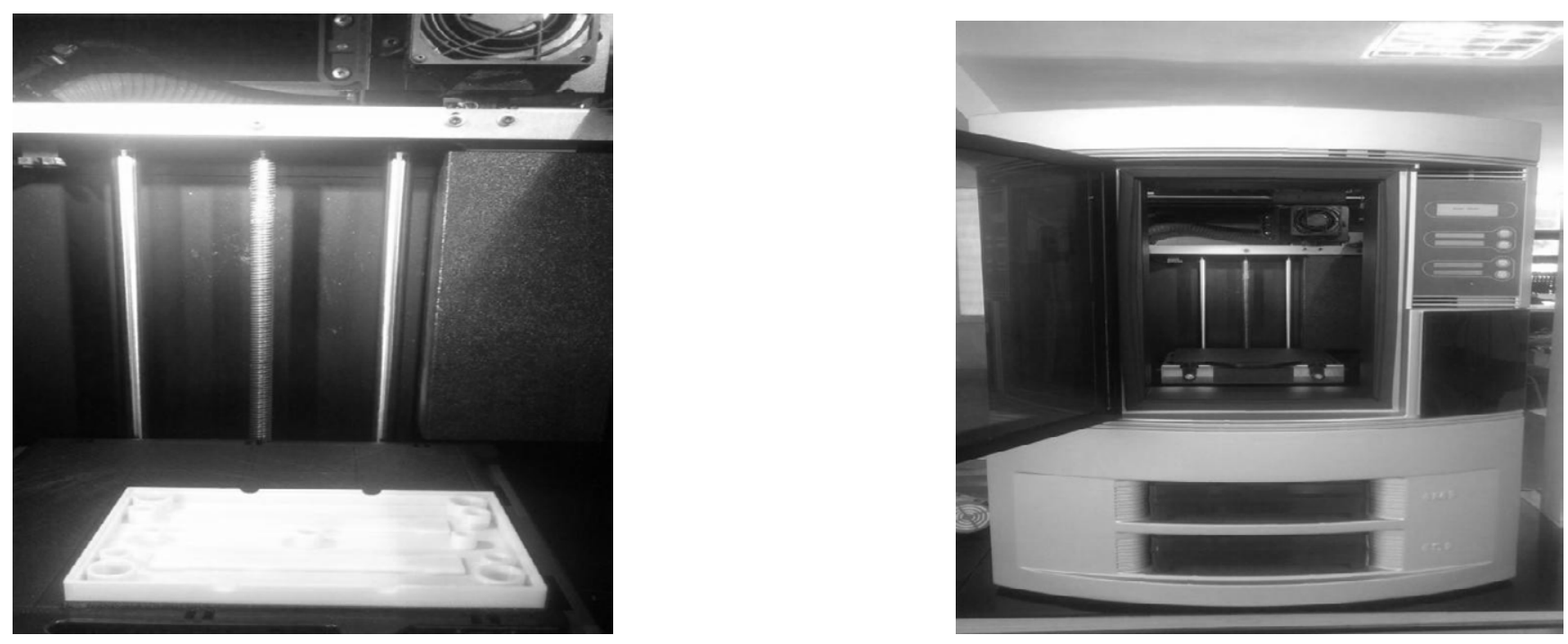

Figura 5. Impresora y piezas impresas.

Fuente: Elaboración propia 


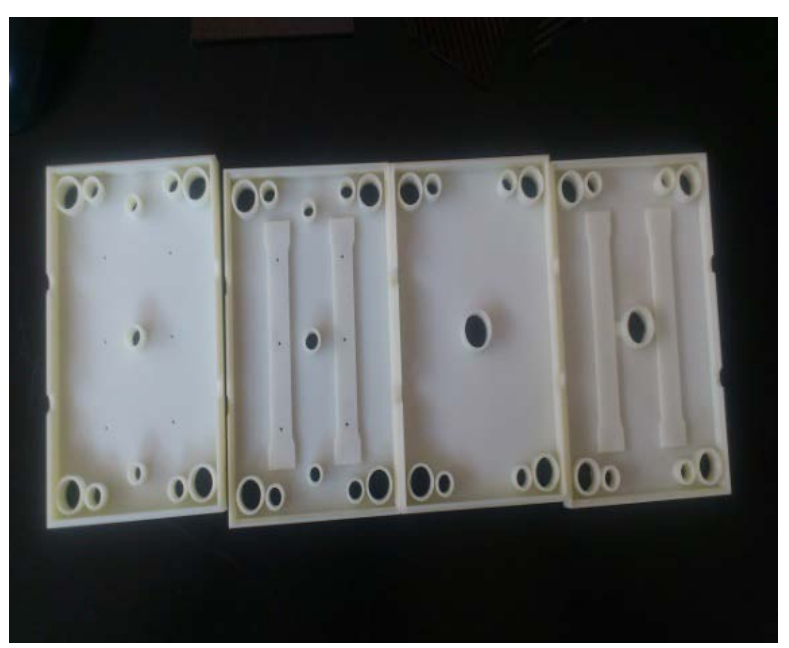

Figura 6. Piezas impresas.

Fuente: Elaboración propia

con el que trabajó la máquina. Como la geometría de las piezas no era compleja, no hubo necesidad de pasar las piezas por el solvente (figura 6).

\section{RESULTADOS}

Se consiguieron las piezas cavidades del molde de inyección, distribuidas en cuatro partes. Con base en estas piezas se podrá realizar un análisis más exhaustivo de lo que significa el prototipo rápido de piezas realizadas en impresión 3D.

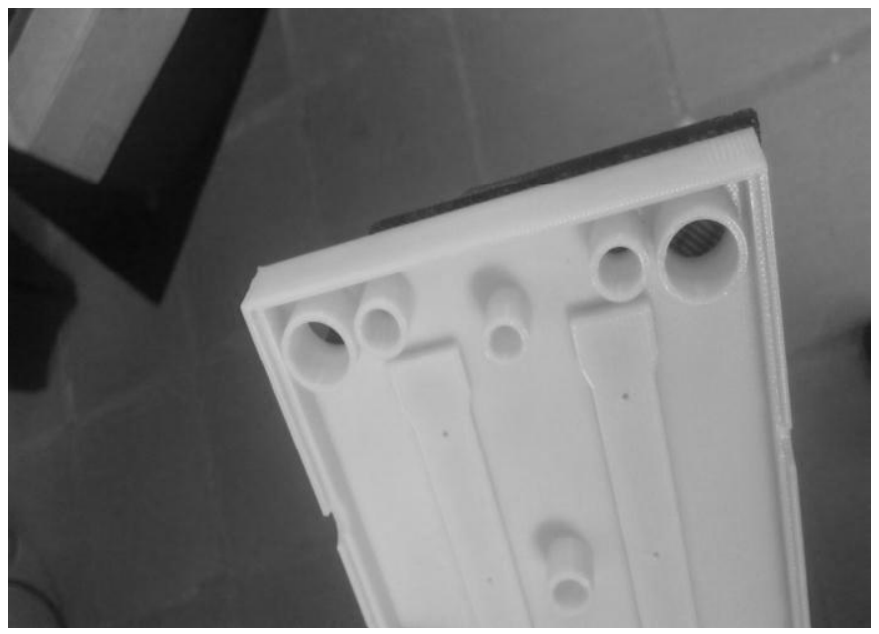

Uno de los fines de un prototipo o modelo es permitir el análisis de sus componentes y observar un comportamiento relativo de lo que se puede esperar y se debe mejorar en el proceso real que se está estudiando y de cada una de las piezas obtenidas.

La figura 7 muestra las dos placas del molde con cavidades. Con base en ellas se puede realizar el estudio de optimización de los canales inyección. Adicionalmente tienen la ventaja de que se

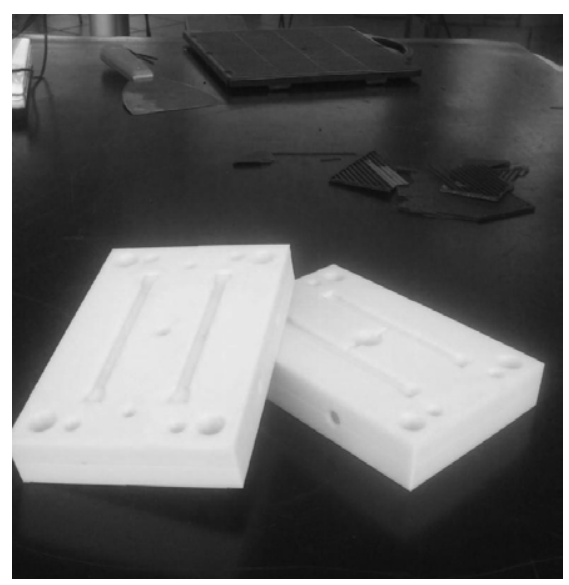

Figura 7. Placas de la cavidad impresas.

Fuente: Elaboración propia 
pueden transportar y manejar gracias a su peso reducido por el material de fabricación.

\section{DISCUSIÓN}

Una vez concluido el proceso de impresión de las piezas, se discutieron los siguientes puntos:

- Diseño óptimo. Lo único que puede llegar a impedir un diseño práctico y óptimo para los moldes de inyección es la geometría de las cavidades de la pieza que se va a fabricar. Se puede tomar un modelo estándar del molde y adaptar la cavidad requerida.

- Prototipos elaborados mediante impresión 3D. En cuanto a la viabilidad de estos prototipos, se requiere una calidad mayor en la impresión, que se puede conseguir aumentando los estándares programados en la impresora utilizada, puesto que sería difícil realizar pruebas con un molde elaborado completamente en ABS mediante la impresión 3D.

- Construcción del molde. Por último, la fabricación de un molde en el metal requerido para su implementación en la industria puede resultar "sencilla" siempre que se cuenten con las herramientas que brinden el apoyo necesario (por ejemplo las máquinas de herramientas $\mathrm{CNC}$ ) y puedan cumplir con las especificaciones que sean solicitadas.

\section{CONCLUSIONES}

A lo largo del proyecto se presentaron inconvenientes que se lograron sortear de la mejor manera, desde el problema con el escáner y las piezas, hasta la solicitud de ahorro de espacio y material por parte de la máquina de impresión 3D. SE consiguió experimentar con las tecnologías CAD, CAM, que abren diversas oportunidades para el diseño, en los materiales y la fabricación. Este tipo de software provee facilidad de manejo, rapidez y fluidez de los bosquejos realizados a lápiz representados estructuralmente en el computador.
En cuanto a la implementación de un molde de inyección realizado por impresión 3D en el proceso de inyección, se afirma que solo serviría de estructura, debido a que las propiedades del material con que se fabricó no cumplen los requerimientos necesarios. Se requiere la adaptación del prototipo; una opción puede ser realizar injertos, tanto en las cavidades como en los canales, de material metálico resistente a las presiones y a las temperaturas a las que se someten durante el proceso de inyección.

Por último, el proceso de impresión 3D es una herramienta para el prototipado rápido de piezas que se deseen estudiar o poner a prueba de una forma rápida y eficiente. Esta herramienta ahorra tiempos de estudio y materiales, sin dejar a un lado la parte económica.

\section{FINANCIACIÓN}

Los autores agradecen el apoyo financiero a la Universidad de Pamplona, y al SENA por las instalaciones del Tecno-parque del SENA en Ocaña, en donde se realizó el estudio y desarrollo del molde.

\section{REFERENCIAS}

Alan, L. \& Nguyen , H. (2014). Automatic generation of mold-piece regions and parting curves for complex CAD models in multi-piece mold design. Computer-Aided Design, 15-28.Alvaro, A. \& Gabriel , P. J. (2012). Álvaro Guarín Grisales. Revista Universidad EAFIT, 53-65.

Ching, P., Ming-Tsan , H., \& Yun-Hsiang , H. (2009). Simulation and experimental study in determining injection molding process parameters for thin-shell plastic parts via design of experiments analysis. Expert Systems with Applications, 0752-10759 .

Dimitrov, D. \& Saxer, M. (2012). Productivity Improvement in Tooling Manufacture through High Speed 5 Axis Machining. 5th CIRP Conference on High Performance Cutting 2012, 277-282.

Eghbal, H. \& Abu Bakar, S. (2012). Analysis of warpage and shrinkage properties of injection-molded micro gears polymer composites using numerical 
simulations assisted by the Taguchi method. Materials and Design, 62-71.

Hugo, R., Binayak, B., Wei , W., \& Ahna, S.-H. (2014). 3D soft lithography: A fabrication process for thermocurable polymers. Journal of Materials Processing Technology, 1-15.

Joanna, N., Karl , W., \& David , D. (2014). Rapid Tooling Injection Molded Prototypes: A Case Study in Artificial Photosynthesis Technology. 6th CIRP International Conference on High Performance Cutting, HPC2014, 251-256.

Mustafa, K., Usuf, K., \& Kamber, O. (2010). Influence of molding conditions on the shrinkage and roundness of injection molded parts. The International Journal of Advanced Manufacturing Technology, 571-578.

Satoshi , K. \& Shinji, N. (2014). Multi-objective optimization of volume shrinkage and clamping force for plastic injection molding via sequential approximate optimization. Simulation Modelling Practice and Theory, 35-44.

Xuan-Phuong, D. (2014). General frameworks for optimization of plastic injection molding process parameters. Simulation Modelling Practice and Theory, 15-27. 
